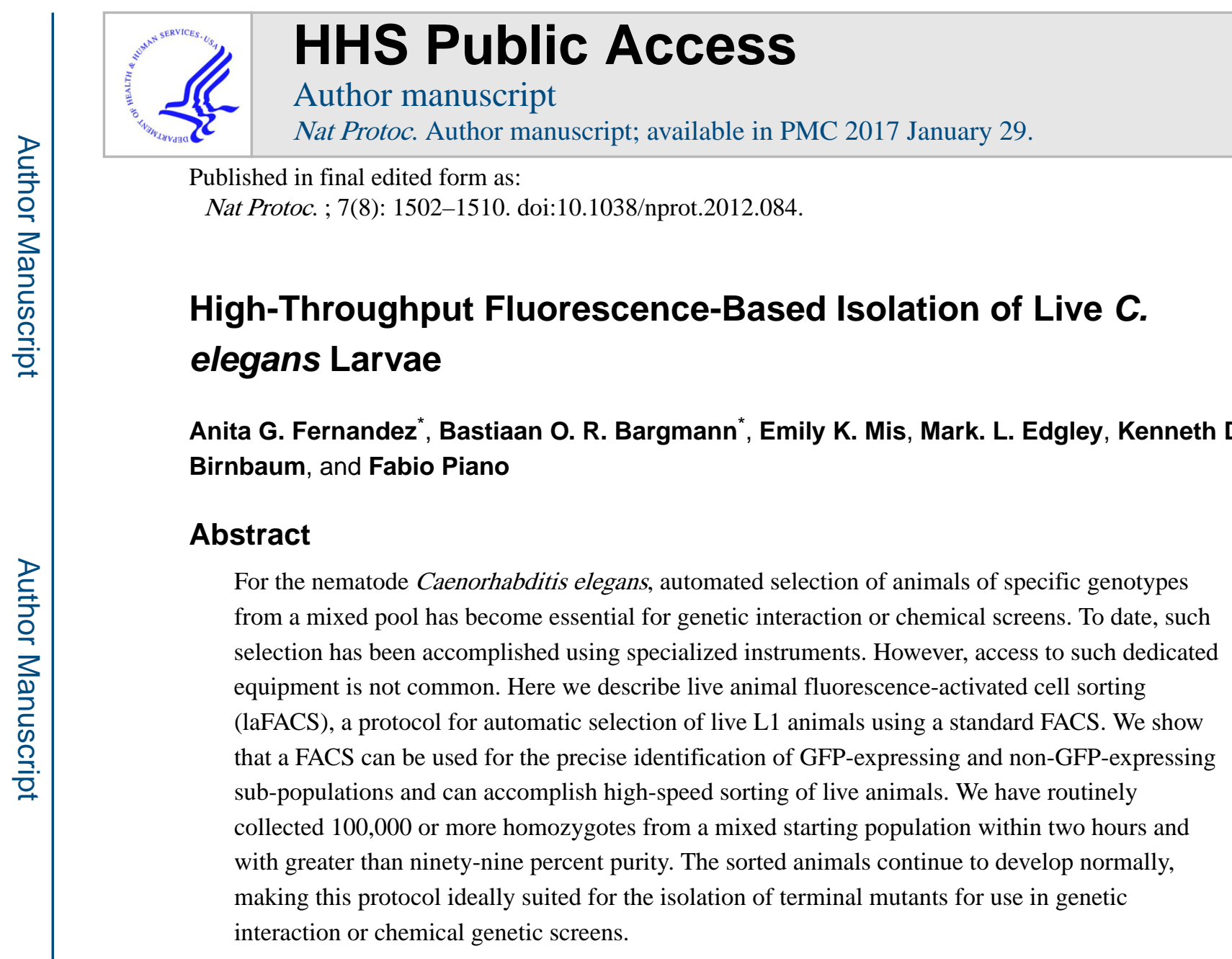

Keywords

C. elegans; worm sorting; FACS; embryonic lethality; maternal-effect lethality; sterility

\title{
INTRODUCTION
}

Caenorhabditis elegans is an important model system for studying the fundamental genetic mechanisms underlying developmental and behavioral biology ${ }^{1}$. Among its many advantages as an animal model system is that it can be easily grown to very large populations and there is a spectacular infrastructure supporting this system that includes mutants affecting over half of its genes $\left({ }^{2}\right.$ and pers. comm. Donald G. Moerman and Robert Waterston), genome-scale RNAi reagents ${ }^{3-5}$, and an extensive collection of green fluorescent protein (GFP)-marked strains ${ }^{6}$. These features make this model system well suited for large-scale studies ${ }^{7-9}$.

CORRESPONDING AUTHORS. Anita Fernandez (AFernandez@fairfield.edu) Bastiaan Bargmann (bbargmann@ucsd.edu) and Fabio Piano (fp1@nyu.edu).

These authors contributed equally AUTHOR CONTRIBUTIONS STATEMENTS

FP and AF initially developed the idea, BB and AF developed the FACS conditions, and FP, AF, BB, EM, ME and KB contributed to the realization of the protocol.

COMPETING FINANCIAL INTERESTS STATEMENT

The authors declare that they have no competing financial interests. 
It is estimated that $30 \%$ of the genes In $C$. elegans have a terminal phenotype such as embryonic lethality, maternal-effect lethality, or sterility ${ }^{10}$. While some terminal phenotypes (e. g. sterility) are viable, they do not produce viable offspring and thus it is impossible to grow them as a pure population of homozygotes. Balancer chromosomes are key tools for preventing the loss of terminal alleles in a segregating population. In a balanced heterozygote, the wild-type allele is carried by a balancer chromosome, which bears chromosomal aberrations such as inversions or translocations that prevent recombination events. Some balancers carry recessive lethal alleles, causing homozygous balancer embryos to be inviable, thus preventing the proliferation of animals that have lost the recessive terminal allele. Many balancers contain GFP transgenes (Table 1), enabling the easy identification of heterozygous animals. Since the balancer chromosome resists recombination, the GFP transgene is always associated with the wild-type allele and thus homozygous mutant individuals can easily be identified due to lack of GFP expression (see Figure 1 for an example of a balanced strain). However, manual isolation of homozygous individuals from a heterogeneous population is too labor intensive to be practicable for large-scale analyses.

The microscopic size of $C$. elegans facilitates the use micro-fluidic and flow cytometric systems to analyze or sort and collect individual animals with specific optical properties. The COPAS (complex object parametric analysis and sorting) biosorter is expert apparatus developed especially for the optical analysis and sorting of nematodes ${ }^{11}$. This specialized sorter is well suited to the fluorescence-based isolations of sub-populations in a heterogeneous batch ${ }^{11}$ and is capable of much more, including stage-specific sorting and analysis of fluorescence within specific regions of the animals ${ }^{11-13}$. However, these dedicated machines are not widely available and therefore accessibility to high-throughput techniques for isolation of sterile/lethal mutants is restricted.

Recently, it has been reported that a fluorescence activated cell sorter (FACS), a more prevalent piece of equipment, can be used for automated sorting of live C. elegans larvae ${ }^{14}$ as well as fixed embryos ${ }^{15}$. We have successfully used this technique, dubbed laFACS (live animal FACS), to isolate over one hundred thousand larvae for RNAi genetic interaction screening of a maternal lethal mutant ${ }^{14}$. We used L1-stage larvae because 1) their small size (about $250 \mu \mathrm{m}$ long and $15 \mu \mathrm{m}$ wide) allows them to run through the FACS fluidics system and 2) starvation arrests growth at this stage and enables easy accumulation of developmentally synchronized animals. We employed the GFP-negative balancer signature to rapidly identify homozygous mutant larvae in a mixed population and sort them to high purity.

Such isolated larvae can be used for genetic interaction screens, chemical genetic screens, or any other application where large numbers of animals of a particular lethal or sterile genotype are required. There could conceivably be additional applications of laFACS. For example, it could be used to screen large populations for reporter gene activation. However, FACS machines and flow cytometers are designed to quantify fluorescence in relatively small particles and will not give a highly accurate quantitative readout of fluorescence in large objects such as $C$. elegans larva. Unlike The COPAS biosorter that is specifically designed to sort worms of all stages based on size and/or degree of fluorescence, we have 
used a FACS only to sort L1 larvae on the basis of presence or absence of fluorescence. Case-by-case trials will have to be undertaken to investigate whether laFACS is suitable for alternative applications. Furthermore, due to size restrictions, the use of laFACS is limited to L1-stage larvae (and embryos). Nonetheless, the ability to use a FACS to sort live $C$. elegans greatly increases the accessibility and feasibility of high throughput analyses in this model organism.

\section{EXPERIMENTAL DESIGN}

\section{Strain construction}

In the example presented here, we used laFACS to collect a pure population of maternaleffect lethal ( $m e l-28^{16,17}$ ) homozygotes that we then subjected to an RNAi-based genetic interaction screen ${ }^{14}$. First, we generated a GFP-balanced mel-28 strain called PF405. In this strain, the mel-28 allele and a cis-linked unc-32 allele were carried on an otherwise wildtype chromosome, and were balanced by the $q C 1$ balancer containing the $q I s 26$ insertion (Figure 1). The $q I s 26$ insertion includes both a lag-2::GFPreporter and the dominant rol-6 allele $s u 1006^{18}$, and gives rise to recessive lethality. PF405 heterozygotes produce homozygous $q C 1$ [qIs 26] animals that die as embryos, heterozygous mel-28 unc-32/ $q C 1$ [qIs 26] animals that have a Rol-6 phenotype and express GFP in the distal tip cells of the gonads, and mel-28 unc-32 homozygotes which are uncoordinated, do not express GFP, and produce only dead eggs (Figures 1 and 2).

Proper strain construction is critical for the successful execution of laFACS. We used a balancer that carries, in addition to the GFP transgene, a dominant visible marker (rol-6(su1006)) and a recessive lethal lesion. The visible marker enables distinction of heterozygotes without need of an epifluorescence microscope. If there are no appropriate balancers with this feature, it is recommended that the terminal allele of interest be kept in cis with a recessive visible marker. In our PF405 strain, the mel-28 chromosome also carries an unc-32 allele, thus mel-28 homozygotes from this strain are always uncoordinated (Figures 1 and 2E).

Using a balancer that confers recessive lethality is preferable, but not required. To grow worms for this protocol, we chose heterozygous animals to start the initial stocks, and then expanded them for two generations without specifically picking heterozygotes. Thus the L1's we sorted were the F2 of the heterozygotes initially picked. Since our balancer was homozygous lethal and mel-28 homozygotes do not produce larvae, all of these L1's arose from a heterozygous carrier and thus one out of three are expected to be mel-28 homozygotes (Figure 1). When using homozygous viable balancers, with each generation there will be balancer homozygotes that have lost the allele of interest. This lowers the expected yield of desired animals homozygous for the recessive terminal allele. However, because this protocol allows for the sorting of hundreds of thousands of animals, it should still be possible to use this protocol to isolate substantial numbers of homozygotes using a balancer that is not homozygous lethal. 


\section{Sorting larvae}

To prepare the larvae for sorting, we used sodium hypochlorite treatment to isolate embryos from PF405 adults and allowed these to hatch without food so the animals were arrested at the L1 stage (Figure 3). After filtering the hatched L1's to remove large debris, we used laFACS to isolate GFP-negative homozygous mel-28 L1 animals from the population. To prime the FACS for larval sorting we installed a $100 \mu \mathrm{m}$ nozzle and we set up a stream at a lower than usual drop-drive frequency ( $\sim 16 \mathrm{kHz}$ as opposed to $\sim 38 \mathrm{kHz}$; see Figure $4 \mathrm{a}$ ) in order to optimize worm viability while passing through the apparatus.

To determine FACS parameters and establish a signature for worms that do not express GFP we ran wild-type (N2) L1's. A gate was set up in a forward scatter versus side scatter plot in order to distinguish worm-sized events from debris. In effect, larvae were so large that they were off-scale (even at the lowest photo multiplier tube voltage settings) and this "worm gate" encompassed only the events with the highest forward scatter values (Figure $4 \mathrm{~b}$ left panel). This gate is especially useful when sorting strains that produce a lot of small debris, e.g. remnants of the inviable embryos present in the mel-28 population (see Figures $3 \mathrm{c}$ and $4 \mathrm{c}$ left panels). Only events that passed the "worm gate" were evaluated for green versus red fluorescent emission (emission around 530 and $610 \mathrm{~nm}$, respectively; Figures $4 \mathrm{~b}$ and c right panels). Voltage settings were adjusted so that GFP-negative larvae showed an equal ratio of green to red (auto)fluorescence and could be distinguished from GFP-positive larvae which showed relatively higher green than red fluorescence (Figures $4 b$ and $c$ ). Gates were set to identify both GFP-positive and GFP-negative populations.

\section{Controls}

Going through the steps of this protocol, it is important to regularly inspect the samples microscopically. We looked for larval viability, genotypic purity (as determined by GFPfluorescence as well as Rol and Unc phenotypic characteristics), debris content and clumping/coagulation. Synchronized and filtered wild-type larvae and a sample of pure GFP-positive larvae can be taken along as a negative- and positive control for the FACS run. These control samples are especially practical when first setting up the technique.

\section{MATERIALS}

\section{REAGENTS}

- C. elegans strain carrying allele of interest balanced by a GFP-containing balancer chromosome.

- OP50 strain of E. coli (Caenorhabditis Genetics Center, http://www.cbs.umn.edu/ $\mathrm{CGC} /$ )

- $\quad$ Nematode Growth Medium (US Biological, cat. no. N1000)

- $\quad$ tryptone (Fisher Scientific cat. no. BP1421)

- $\mathrm{CaCl}_{2}$ dihydrate (Fisher Scientific cat. no. BP510)

- $\mathrm{KH}_{2} \mathrm{PO}_{4}$ (Fisher Scientific cat. no. BP362) 
- $\quad \mathrm{K}_{2} \mathrm{HPO}_{4}$ (Fisher Scientific cat. no. BP363)

- $\mathrm{Na}_{2} \mathrm{HPO}_{4}$ heptahydrate (Fisher Scientific cat. no. BP331)

- $\quad \mathrm{NaCl}$ (Fisher Scientific cat. no. BP358)

- $\quad \mathrm{MgSO}_{4}$ heptahydrate (Fisher Scientific cat. no. BP213)

- $\quad \mathrm{NaOH}$ (Fisher Scientific cat. no. BP359)

- $\quad$ sucrose (Fisher Scientific cat. no. BP220)

- household sodium hypochlorite solution (4-6\%, w/v)

- $\quad$ PBS (phosphate buffered saline, $\mathrm{pH7.0)}$

- $\quad 100$ mm plates (Fisher Scientific cat. no. 08-757-12)

- $\quad 150 \mathrm{~mm}$ plates (Fisher Scientific cat. no. 08-757-14)

- $\quad$ Cell strainer (40 $\mu \mathrm{m}$; BD Falcon, cat. no. 352340)

- $\quad 50 \mathrm{ml}$ conical tubes (Fisher Scientific cat. no. 14-432-22)

- $\quad 15 \mathrm{ml}$ collection tubes (Fisher Scientific cat. no. 05-527-90)

\section{REAGENT SETUP}

$100 \mathrm{~mm}$ standard NGM plates-Prepare $1 \mathrm{M} \mathrm{CaCl}_{2}$ and $1 \mathrm{M} \mathrm{MgSO}_{4}$. Autoclave and allow to cool to room temperature. Prepare $1 \mathrm{M} \mathrm{K}_{2} \mathrm{HPO}_{4}$ (dibasic) and $1 \mathrm{M} \mathrm{KH}_{2} \mathrm{PO}_{4}$ (monobasic). Autoclave and allow to cool to room temperature. Prepare $1 \mathrm{M}$ Potassium Phosphate buffer (pH 6.0) by mixing $132 \mathrm{ml}$ of autoclaved $1 \mathrm{M} \mathrm{K}_{2} \mathrm{HPO}_{4}$ with $868 \mathrm{ml}$ of autoclaved $1 \mathrm{M} \mathrm{KH}_{2} \mathrm{PO}_{4}$ in a sterile bottle.

For one liter of NGM, mix $23.005 \mathrm{~g}$ of Nematode Growth Medium in $975 \mathrm{ml}$ of deionized water. Autoclave with a stir bar in the solution. After removal from the autoclave, stir the molten solution on a stir plate until it has cooled to $\sim 55^{\circ} \mathrm{C}$, then add $24.7 \mathrm{ml}$ of $1 \mathrm{M}$ Potassium Phosphate solution, $1 \mathrm{ml}$ of $1 \mathrm{M} \mathrm{CaCl}_{2}$, and $1 \mathrm{ml}$ of $1 \mathrm{M} \mathrm{MgSO}_{4}$. After mixing, pour $28 \mathrm{ml}$ per each $100 \mathrm{~mm}$ plate. If pouring by hand, plan to get approximately 30 plates per liter. Allow plates to rest at room temperature for 1-3 days, to allow condensation to evaporate. Unseeded NGM plates may be stored tightly sealed in plastic sleeves and kept at $4^{\circ} \mathrm{C}$ for months. To seed plates, grow a $50 \mathrm{ml}$ culture of OP 50 E. coli in LB without antibiotics using standard sterile microbiological techniques. This culture may be stored at $4^{\circ} \mathrm{C}$ for four weeks. Drop $200 \mu \mathrm{l}$ of OP50 culture onto each poured NGM plate, and allow to rest at room temperature overnight. Seeded plates may be stored at $4^{\circ} \mathrm{C}$ for four weeks.

$150 \mathrm{~mm}$ superseeded $5 \times$ peptone plates-For one liter of NGM, mix $23.005 \mathrm{~g}$ of Nematode Growth Medium and $10 \mathrm{~g}$ of tryptone in $975 \mathrm{ml}$ of deionized water. The tryptone will not go into solution completely until autoclaved. Autoclave with a stir bar in the solution. After removal from the autoclave, stir the molten solution on a stir plate until it has cooled to $\sim 55^{\circ} \mathrm{C}$, then add $24.7 \mathrm{ml}$ of $1 \mathrm{M}$ Potassium Phosphate solution, $1 \mathrm{ml}$ of $1 \mathrm{M}$ $\mathrm{CaCl}_{2}$, and $1 \mathrm{ml}$ of $1 \mathrm{M} \mathrm{MgSO}_{4}$ (solutions described in standard NGM recipe). Pour $\sim 55 \mathrm{ml}$ per $150 \mathrm{~mm}$ plate, if pouring by hand expect to get 18 plates per liter. Let plates sit at room 
temperature for 1-3 days, to allow condensation to evaporate. Unseeded plates may be stored tightly sealed in plastic sleeves and kept at $4^{\circ} \mathrm{C}$ for months. To superseed the plates, prepare a culture of OP-50 in LB, then pellet it and resuspend it in $1 / 10^{\text {th }}$ the volume of M9 (e.g., a $200 \mathrm{ml}$ solution of OP50 would be pelleted and resuspended into $20 \mathrm{ml}$ of M9). Use a sterile bent glass rod to spread $\sim 1 \mathrm{ml}$ of the concentrated OP50 across each $150 \mathrm{~mm} 5 \times$ peptone plate, making sure the bacteria covers the surface area of the plate. Seeded plates may be stored at $4^{\circ} \mathrm{C}$ for four weeks.

M9-Mix $3.0 \mathrm{~g}$ of $\mathrm{KH}_{2} \mathrm{PO}_{4}, 6.0 \mathrm{~g}$ of $\mathrm{Na}_{2} \mathrm{HPO}_{4}$, and $5.0 \mathrm{~g}$ of $\mathrm{NaCl}$ in 1 liter of deionized $\mathrm{H}_{2} \mathrm{O}$. Autoclave, then cool to room temperature. Using sterile technique, add $1 \mathrm{ml}$ of sterile $1 \mathrm{M} \mathrm{MgSO}_{4}$ and swirl to mix. Prepared M9 may be stored at room temperature indefinitely.

Sodium Hypochlorite Solution-Mix $30 \mathrm{ml}$ of household household sodium hypochlorite solution (bleach) with $60 \mathrm{ml}$ deionized $\mathrm{H}_{2} \mathrm{O}$ and $10 \mathrm{ml} 10 \mathrm{~N} \mathrm{NaOH}$. (Store at room temperature in the dark for no more than one month.)

CAUTION: hypochlorite solutions lose activity over time. Use proper ventilation when working with sodium hypochlorite.

\section{EQUIPMENT}

- $\quad$ FACS equipped with a $488 \mathrm{~nm}$ excitation laser and 530/30 nm and 610/20 nm emission filters (BD FACSAria I)

- Epifluorescence dissecting microscope suitable for imaging GFP (such as the Leica MZ16FA).

\section{EQUIPMENT SETUP}

FACS preparation-Clean and sterilize FACS fluidics system, if required, and use PBS as sheath fluid. Install a $100 \mu \mathrm{m}$ nozzle and set the sheath pressure at 20 p.s.i. Set up a stable stream with low drop-drive frequency. Standard operating parameters for the FACSAria run FACS drop-drive frequency in the vicinity of $38 \mathrm{kHz}$ when using the $100 \mu \mathrm{m}$ nozzle, thereby creating a stable droplet break-off point at the correct distance from the nozzle. However, at this frequency we noted a low recovery rate $(+/-50 \%)$, indicating a large proportion of the larvae did not survive the sort. A stable break-off point can be obtained at lower frequency $(+/-16 \mathrm{kHz} ;$ Figure 4a). At this lower frequency it is also possible to set an accurate drop delay and stable side streams (as determined by Accudrop beads, BD Biosciences). Sorting with this setup led to a greatly improved recovery rate $(+/-80 \%)$. Set the sample agitation function (if available) to ensure larvae don't settle to the bottom of the sample tube during the sort.

Run a wild-type control (Figure 4b). Adjust the flow rate to get to approximately 100-300 events per second. Prepare a scatter-plot analysis of forward scatter area (FSC-A) versus side scatter area (SSC-A) (Figure 4b left panel). Set low power to the FSC photo multiplier tube (PTM); intact L1 larvae will give a FSC-A signal that is off-scale, debris will give relatively weaker signals. Set SSC-A PMT power to visualize the widest possible range of events. This scatter plot will make it possible to monitor debris content in your sample (Figures $4 \mathrm{~b}$ and $\mathrm{c}$ 
left panels). Set a gate to isolate events with the highest FSC-A signal, these contain the larvae (Figure 4b left panel "worm gate"). Prepare a scatter-plot analysis of green fluorescence (GFP; $488 \mathrm{~nm}$ excitation and emission at 530/30 nm) and red fluorescence (red spectrum autofluorescence [RSA]; $488 \mathrm{~nm}$ excitation and emission at 610/20 nm) and display only the population that passes the worm gate set in the FSC-A vs. SSC-A plot (Figure $4 \mathrm{~b}$ right panel). Adjust the GFP and RSA PMT settings to center the event population representing the GFP-negative, intact larvae (Figure $4 \mathrm{~b}$ right panel). This scatter plot will make it possible to distinguish true GFP fluorescence from autofluorescence by determining the ratio of green to red fluorescence. Use compensation settings to adjust for spectral overlap between GFP emission and the RSA filter set (settings will depend on the equipment and reporter strain used). This will aid in the distinction between GFP-positive and GFP-negative populations.

Prepare a $15 \mathrm{ml}$ collection tube in the sort block. The collected larvae will remain viable in the PBS that accumulates in the collection tube throughout the sort. Trial sorts of negativeand positive-control samples should be performed and inspected microscopically to assess recovery rate, viability and purity of the sorted animals.

\section{PROCEDURE}

1. Place 12 GFP-balanced heterozygous hermaphrodite young adults on each of 10 $100 \mathrm{~mm}$ standard NGM plates seeded with OP50 and allow to grow at $20^{\circ} \mathrm{C}$ until plate is full of young adults but worms are not starved (there is OP50 remaining on the plate).

2. Wash worms from plates using $\mathrm{M} 9$ (1-3 $\mathrm{ml}$ per plate) and collect M9 worm suspension to a $50 \mathrm{ml}$ conical tube.

3. Spin collected worms at $700 \mathrm{~g}$ for 5 minutes and carefully decant supernatant.

4. Add $25 \mathrm{ml}$ of cold $\mathrm{M} 9$ and $25 \mathrm{ml}$ of cold $60 \%$ sucrose to worm pellet and mix. (Store autoclaved solutions of M9 and $60 \%$ sucrose at $4^{\circ} \mathrm{C}$ for this purpose.)

5. Spin at $1500 \mathrm{~g}$ at $4^{\circ} \mathrm{C}$ for 5 minutes.

6. Remove gravid worms from the top and place in a new $50 \mathrm{ml}$ conical tube.

7. Wash collected worms with M9 by filling tube with M9, spinning at $700 \mathrm{~g}$ for 5 minutes, then carefully decanting supernatant.

8. Resuspend worm pellet in $\sim 25 \mathrm{ml}$ of sodium hypochlorite solution.

9. Swirl the solution every minute or so. After about 4 minutes, the solution should have become cloudy, indicating that many adults have dissolved. Immediately fill the tube with M9 and spin at $1500 \mathrm{~g}$ at room temperature for 2 minutes. Carefully decant bleach solution without disturbing the worm embryo pellet.

10. Wash the embryo pellet. Fill the tube with M9 and vortex to resuspend pellet, spin at $1500 \mathrm{~g}$ for 2 minutes, and decant M9.

11. Repeat step 10 two more times. 
12. Resuspend washed embryos is $5-10 \mathrm{ml}$ of $\mathrm{M} 9$.

13. Allow collected embryos to hatch in $\mathrm{M} 9$ by rotating at $25^{\circ} \mathrm{C}$ for $14-20$ hours.

14. Plate synchronized larvae to $150 \mathrm{~mm} 5 \times$ peptone plates superseeded with OP50. Place 25,000-50,000 L1 larvae on each plate. Incubate at $20^{\circ} \mathrm{C}$ until most plated worms are gravid adults (3-6 days, depending on the strain). There should be plenty of embryos present on the plate at the time of harvest. ? TROUBLESHOOTING

15. Although the population will be mostly synchronized at this step, purify it further by performing a sucrose float (as in steps 2-7) to separate gravid adults from any remaining larvae. Use one $50 \mathrm{ml}$ conical per five full plates of worms.

16. Treat collected worms with sodium hypochlorite solution to isolate embryos (as in steps 8-11). CRITICAL STEP: The embryos from this step will hatch to form the L1's that will be sorted the following day, so it is important for these embryos to be as clean as possible to avoid putting extra particulate matter through the FACS. Ideally at this stage the adult worm bodies are dissolved completely by the bleach leaving only a pure solution of embryos. However, overbleaching at this step will kill embryos, lowering the L1 yield. Different bottles of bleach have different potencies, thus the amount of time spent bleaching these worms must be determined empirically. After each minute in bleach solution, mix the worm suspension and remove one microliter to examine using a stereoscopic microscope. When $75 \%-90 \%$ of the gravid adults have burst open and released their embryos (Figure 3a), proceed to M9 washes.

17. Allow collected embryos to hatch in at least $5-10 \mathrm{ml}$ of $\mathrm{M} 9$ in a $50 \mathrm{ml}$ conical while rotating at 25 degrees for 14-20 hours (Figure 3b).

18. Dilute the L1's by filling each conical to the top with M9 and filter each tube of L1's $2 \times$ using a $40 \mu \mathrm{M}$ nylon cell strainer (Figure 3c).

19. Concentrate the larvae by spinning at $750 \mathrm{~g}$ for 5 minutes and then resuspend them in M9 to a concentration of 200-300 L1's/ $\mu$ l. Transfer the larval solution to a tube suitable for loading onto the FACS.

20. Inspect the suspension microscopically and examine debris content, viability and purity.

21. Run sample through the FACS and adjust flow rate to approximately 100-300 events per second. ?TROUBLESHOOTING

22. Set gates encompassing the GFP-positive and GFP-negative populations (Figures $4 \mathrm{~b}$ and c).Larvae with GFP expression will form a population of events off the diagonal in the GFP vs. RSA plot (with a high GFP to RSA ratio) not seen in the wild-type control sample (Figures $4 \mathrm{~b}$ and c). ?TROUBLESHOOTING

23. Sort the GFP-negative larvae into a $15 \mathrm{ml}$ collection tube.

24. Concentrate the sorted larvae by spinning at $750 \mathrm{~g}$ for 5 minutes and resuspend them in PBS to a concentration of 200-300 L1's/ $\mu$ l. 
25. Inspect the suspension microscopically and examine recovery-rate, viability and purity. ?TROUBLESHOOTING

26. Re-sort the larval suspension until sufficient purity is obtained (repeat steps 2123 , Figure $4 \mathrm{c}$ middle panels).

27. Cytometrically re-analyze a portion of the final sort to verify purity (Figure 4c lower panels) and inspect the suspension microscopically and examine recoveryrate, viability and purity.

28. Concentrate the larvae by spinning at $750 \mathrm{~g}$ for 5 minutes and resuspend in a medium suitable for the final application. (We used the sorted homozygous mutants in an RNAi-based genetic interaction screen performed in 96-well plates $\left.{ }^{14}\right)$.

29. Clean and sterilize the FACS fluidics system.

\section{TIMING}

The total timing of the protocol varies depending on the strain used, ranging from one week to two weeks. After a sufficient population of the strain has been grown and the L1's isolated, FACS set-up takes 30 minutes and the actual sort takes about 2 hours.

Step 1: 3-6 days

Steps 2-12: 2 hours

Step 13: 14-20 hours

Step 14: 3-6 days

Steps 15-16: 2 hours

Step 17: 14-20 hours

Steps 18-20: 1 hour

Steps 21-29: 2 hours

\section{TROUBLESHOOTING}

\begin{tabular}{|l|l|l|l|}
\hline step & problem & possible reason & solution \\
\hline 14 & $\begin{array}{l}\text { Many eggs do } \\
\text { not hatch }\end{array}$ & $\begin{array}{l}\text { 1. Did not allow enough time } \\
\text { to hatch }\end{array}$ & $\begin{array}{l}\text { 1. Allow longer time for eggs to hatch, some } \\
\text { 2. Overbleaching } \\
\text { strains might require a longer incubation. }\end{array}$ \\
& $\begin{array}{l}\text { 3. Not enough M9 was added } \\
\text { or tube was not agitated. } \\
\text { 4. Balancer itself gives rise to } \\
\text { embryonic lethality }\end{array}$ & $\begin{array}{l}\text { 3. Use at least } 5 \mathrm{ml} \text { of M9, have worms in a } 50 \\
\text { ml conical, and be sure they are rotating } \\
\text { during the entire incubation. }\end{array}$ \\
& $\begin{array}{l}\text { 4. Homozygous lethal balancers and reciprocal } \\
\text { translocation balancers produce some inviable } \\
\text { embryos. Some proportion of embryos will not } \\
\text { hatch, and this should not disrupt further } \\
\text { applications provided there are enough live }\end{array}$
\end{tabular}




\begin{tabular}{|c|c|c|c|}
\hline step & problem & possible reason & solution \\
\hline & & & L1's left for sorting. \\
\hline 21 & $\begin{array}{l}\text { Recurrent } \\
\text { clogged nozzle } \\
\text { on the FACS }\end{array}$ & $\begin{array}{l}\text { 1. Did not bleach stock well } \\
\text { enough, leading to excess of } \\
\text { debris } \\
\text { 2. Filtering insufficient } \\
\text { 3. Worm suspension too } \\
\text { concentrated }\end{array}$ & $\begin{array}{l}\text { 1. Bleach for a longer amount of time to } \\
\text { reduce debris from adult worm bodies. } \\
\text { 2. Dilute worm solution more before filtering } \\
\text { or filter an additional time. } \\
\text { 3. Dilute worm suspension. }\end{array}$ \\
\hline 22 & $\begin{array}{l}\text { No clearly } \\
\text { distinct GFP- } \\
\text { negative } \\
\text { population } \\
\text { visible }\end{array}$ & $\begin{array}{l}\text { 1. Samples with high debris } \\
\text { content will have more debris } \\
\text { that passes through the FSC vs } \\
\text { SSC worm gate (Figure 4c left } \\
\text { panels). Such samples will } \\
\text { display events the GFP vs. RSA } \\
\text { plot with a wide range of } \\
\text { autofluorescent intensity, } \\
\text { visible as a diagonal smear } \\
\text { obscuring the GFP-negative } \\
\text { population (Figure 4c right } \\
\text { panels) }\end{array}$ & $\begin{array}{l}\text { 1. Use the upper and lower limits of RSA- } \\
\text { intensity of the GFP-positive gate to guide the } \\
\text { placement of the GFP-negative gate (Figure } 4 c \\
\text { right panels). }\end{array}$ \\
\hline 25 & $\begin{array}{l}\text { Collected GFP- } \\
\text { larvae do not } \\
\text { have desired } \\
\text { genotype }\end{array}$ & 1. Balancer unstable & $\begin{array}{l}\text { 1. Check GFP- animals from balanced stock to } \\
\text { determine if they have the desired genotype. } \\
\text { If not, the balancer may have broken down } \\
\text { and permitted recombination. Thaw a frozen } \\
\text { stock of the strain to recover the original } \\
\text { balanced line. If this is a recurrent problem, } \\
\text { then try a different balancer. }\end{array}$ \\
\hline 25 & $\begin{array}{l}\text { Some inviable } \\
\text { larvae } \\
\text { recovered }\end{array}$ & $\begin{array}{l}\text { 1. Reciprocal translocation } \\
\text { balancer used }\end{array}$ & $\begin{array}{l}\text { 1. In GFP-marked reciprocal translocation } \\
\text { balancers the GFP insertion is present on just } \\
\text { one of the translocated chromosome arms. } \\
\text { Depending on the location of the terminal } \\
\text { allele, there could be GFP- half-translocation } \\
\text { (aneuploid) animals that are not homozygous } \\
\text { for the terminal allele. If the recovered } \\
\text { animals are to be used for a chemical or } \\
\text { genetic screen, then the presence of some } \\
\text { GFP- inviable larvae of the wrong genotype } \\
\text { should not disrupt the screen provided there } \\
\text { are enough viable larvae collected. To avoid } \\
\text { aneuploidy, use a balancer that is not a } \\
\text { reciprocal translocation. }\end{array}$ \\
\hline
\end{tabular}

\section{ANTICIPATED RESULTS}

We generally achieved an $80 \%$ or greater recovery rate (larvae recovered/events sorted). The unrecovered fraction of larvae disintegrates during the sort and is seen in subsequent re-sorts as debris (Figure 4c). Sorted larvae were viable and showed no reduced growth or survival in subsequent growth assays (compared to an unsorted control; data not shown). The purity of the sorted larvae (GFP-negative larvae/total larvae) was generally $90 \%$ or higher after the initial FACS run in our example sort of mel-28 larvae (this will depend on the initial ratio of GFP-positive to GFP-negative larvae and debris content). A subsequent re-sort of the collected larvae typically gave a final purity of >99\% GFP-negative larvae (Figure 4c).

In order to ensure a yield of at least 100,000 pure, homozygous mel-28 larvae we started out with 660,000 larvae. With the PF405 strain, one in three of the prepared L1 larvae are homozygous mutants and therefore GFP-negative ( 220,000 larvae). The expected proportion of homozygous larvae will vary depending on the balancer used. With an $80 \%$ recovery rate ( 176,000 successfully sorted larvae) and 90\% purity ( 158,000 GFP-negative larvae) this first sort was re-sorted to obtain $~ 127,000$ GFP-negative larvae with $>99 \%$ 
purity. This isolation required one week of growth and larval preparation time and 3 hours of reserved FACS time. We used a FACSAria I (BD Biosciences) in a core facility shared by many other users and none of the subsequent applications were affected by sorting $C$. elegans larvae.

\section{Acknowledgments}

We thank Erik Andersen, Donald G. Moerman, and Robert Waterston for sharing unpublished data, Pui-leng Ip, Jessica Lucas and Katherine Erikson for technical assistance, and Scott D. Weatherbee for critically reviewing the manuscript. Funding sources included the NICHD (R01HD046236) and NHGRI (U01 HG004276) to FP, the NIH (R01GM078279-01) to KDB, and the NSF (0827858) and Fairfield University start-up funds to AGF. Nematode strains were provided by the Caenorhabditis Genetics Center, which is funded by the NIH National Center for Research Resources (NCRR).

\section{REFERENCES}

1. Brenner S. The genetics of Caenorhabditis elegans. Genetics. 1974; 77(1):71-94. [PubMed: 4366476]

2. Moerman DG, Barstead RJ. Towards a mutation in every gene in Caenorhabditis elegans. Briefings in functional genomics \& proteomics. 2008; 7(3):195-204. [PubMed: 18417533]

3. Kamath RS, et al. Systematic functional analysis of the Caenorhabditis elegans genome using RNAi. Nature. 2003; 421(6920):231-237. [PubMed: 12529635]

4. Reboul J, et al. C. elegans ORFeome version 1.1: experimental verification of the genome annotation and resource for proteome-scale protein expression. Nat Genet. 2003; 34(1):35-41. [PubMed: 12679813]

5. Lamesch P, et al. C. elegans ORFeome version 3.1: increasing the coverage of ORFeome resources with improved gene predictions. Genome research. 2004; 14(10B):2064-2069. [PubMed: 15489327]

6. Mohler WA, Isaacson AB. Imaging embryonic development in Caenorhabditis elegans. Cold Spring Harbor protocols. 2010(3) pdb top71.

7. Gunsalus KC, et al. Predictive models of molecular machines involved in Caenorhabditis elegans early embryogenesis. Nature. 2005; 436(7052):861-865. [PubMed: 16094371]

8. Sönnichsen B, et al. Full-genome RNAi profiling of early embryogenesis in Caenorhabditis elegans. Nature. 2005; 434(7032):462-469. [PubMed: 15791247]

9. Green RA, et al. A High-Resolution C. elegans Essential Gene Network Based on Phenotypic Profiling of a Complex Tissue. Cell. 2011; 145(3):470-482. [PubMed: 21529718]

10. Kemphues, K. The $C$. elegans Research Community. WormBook.

11. Pulak R. Techniques for analysis, sorting, and dispensing of C. elegans on the COPAS flow-sorting system. Methods in molecular biology (Clifton, N.J. 2006; 351:275-286.

12. Dupuy D, et al. Genome-scale analysis of in vivo spatiotemporal promoter activity in Caenorhabditis elegans. Nature biotechnology. 2007; 25(6):663-668.

13. Doitsidou M, Flames N, Lee AC, Boyanov A, Hobert O. Automated screening for mutants affecting dopaminergic-neuron specification in C. elegans. Nature methods. 2008; 5(10):869-872. [PubMed: 18758453]

14. Fernandez AG, Mis EK, Bargmann BO, Birnbaum KD, Piano F. Automated sorting of live $C$. elegans using laFACS. Nature methods. 2010; 7(6):417-418. [PubMed: 20436474]

15. Stoeckius M, et al. Large-scale sorting of C. elegans embryos reveals the dynamics of small RNA expression. Nature methods. 2009; 6(10):745-751. [PubMed: 19734907]

16. Fernandez AG, Piano F. MEL-28 is downstream of the Ran cycle and is required for nuclearenvelope function and chromatin maintenance. Curr Biol. 2006; 16(17):1757-1763. [PubMed: 16950115] 
17. Galy V, Askjaer P, Franz C, Lopez-Iglesias C, Mattaj IW. MEL-28, a novel nuclear-envelope and kinetochore protein essential for zygotic nuclear-envelope assembly in C. elegans. Curr Biol. 2006; 16(17):1748-1756. [PubMed: 16950114]

18. Mello CC, Kramer JM, Stinchcomb D, Ambros V. Efficient gene transfer in C. elegans: extrachromosomal maintenance and integration of transforming sequences. The EMBO journal. 1991; 10(12):3959-3970. [PubMed: 1935914]

19. Edgley ML, Riddle DL. LG II balancer chromosomes in Caenorhabditis elegans: mT1(II;III) and the mIn1 set of dominantly and recessively marked inversions. Mol Genet Genomics. 2001; 266(3):385-395. [PubMed: 11713668]

20. Edgley MK, Baillie David L, Riddle Donald L, Rose Ann M. WormBook. 2006 The C. elegans research community. 
F1

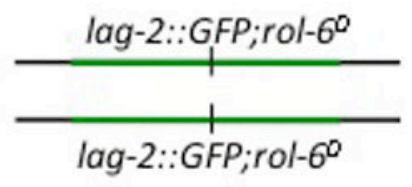

$1 / 4$ inviable

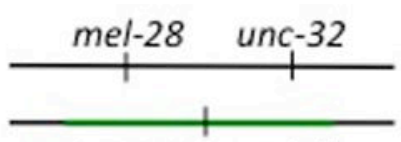

lag-2::GFP;rol-60

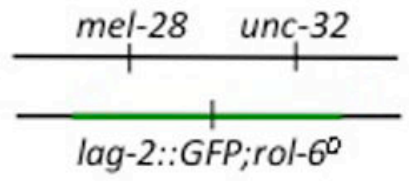

$1 / 2$ roller; GFP+
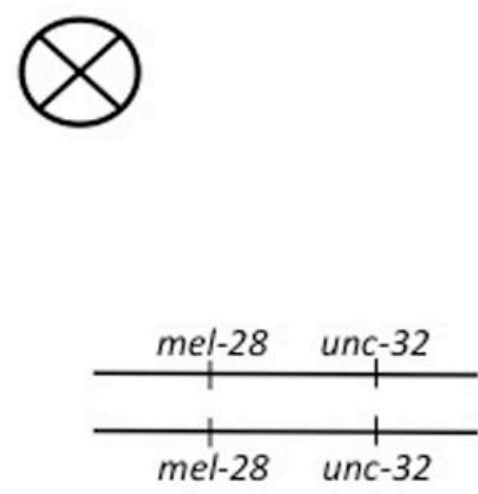

$1 / 4$ unc; GFP-; mel-28

Figure 1. Strain PF405 Genetics

The $q C 1$-balanced region of chromosome is shown in green. We used a version of $q C 1$ with the $q I s 26$ insertion that has the lag-2::GFP transgene and the dominant rol-6 allele su1006. The $q I s 26$ insertion site created a recessive lethal allele such that $q C 1$ [qIs26] homozygotes are inviable. Heterozygotes have the Rol-6 phenotype and express GFP. Animals that have lost the balancer do not express GFP, are uncoordinated, and are mel-28 (and thus produce only dead embryos). 

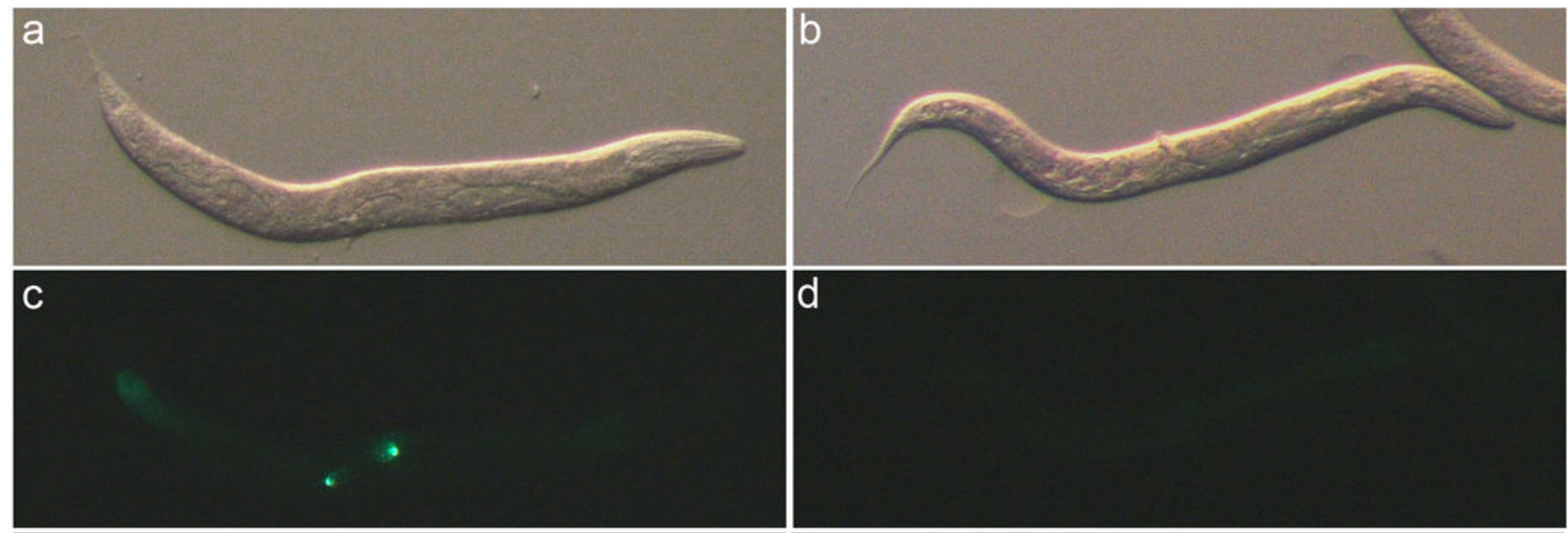

e

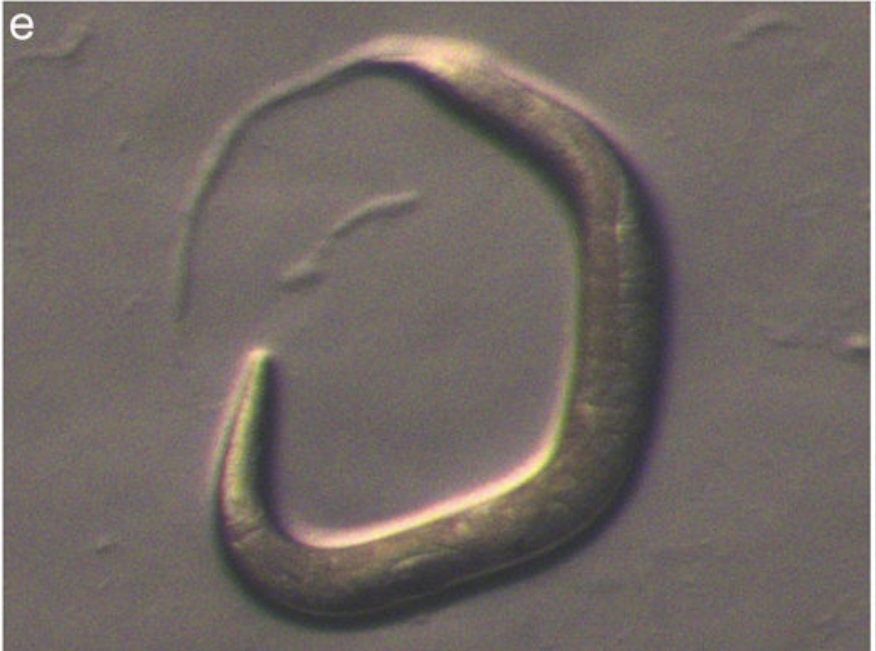

Figure 2. Strain PF405 Phenotypes

(a, c, e) Heterozygous mel-28 unc-32/qC1[qIs26] adults (Rol-6 phenotype). (b, d, f) Homozygous mel-28 unc-32 adults from the PF405 strain. Images were captured in brightfield (a, b, d, f), and with GFP filters (c-d). (a-d) Animals were anesthetized with levamisole and immobilized on an agar pad. (e-f) characteristic behavior of Rol-6 (e) and Unc (f) adults on an NGM plate . 

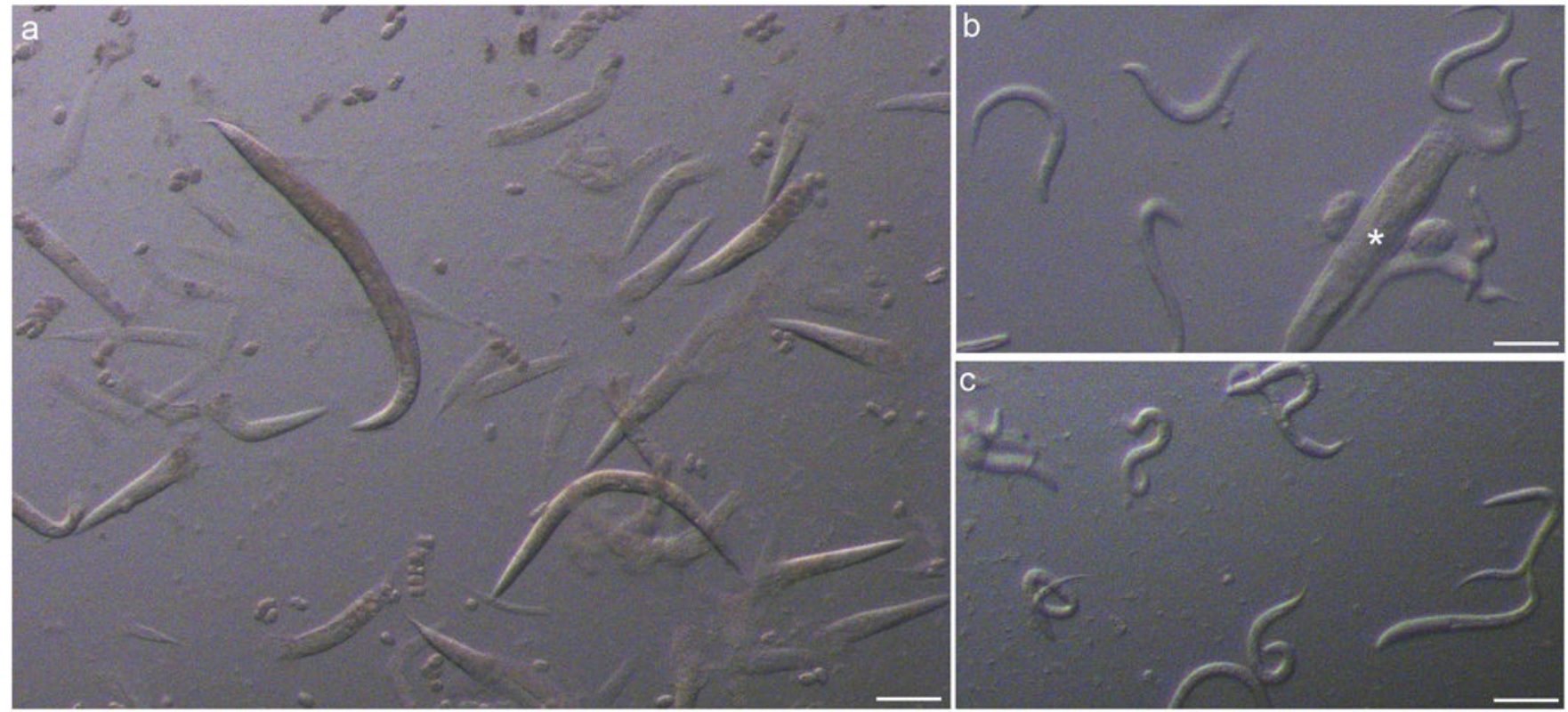

Figure 3. Preparation of C. elegans for laFACS

(a) After a $\sim 4$ minute treatment with sodium hypochlorite and agitation, most adults have burst and freed their embryos (arrowhead). (b) After overnight incubation in M9, many L1 larvae have hatched but large segments of adult corpses (asterisk) remain. (c) After filtering, only small debris and L1 larvae remain. Scale bar $=200 \mu \mathrm{m}(\mathbf{a}) .100 \mu \mathrm{m}(\mathbf{b}-\mathbf{c})$. 

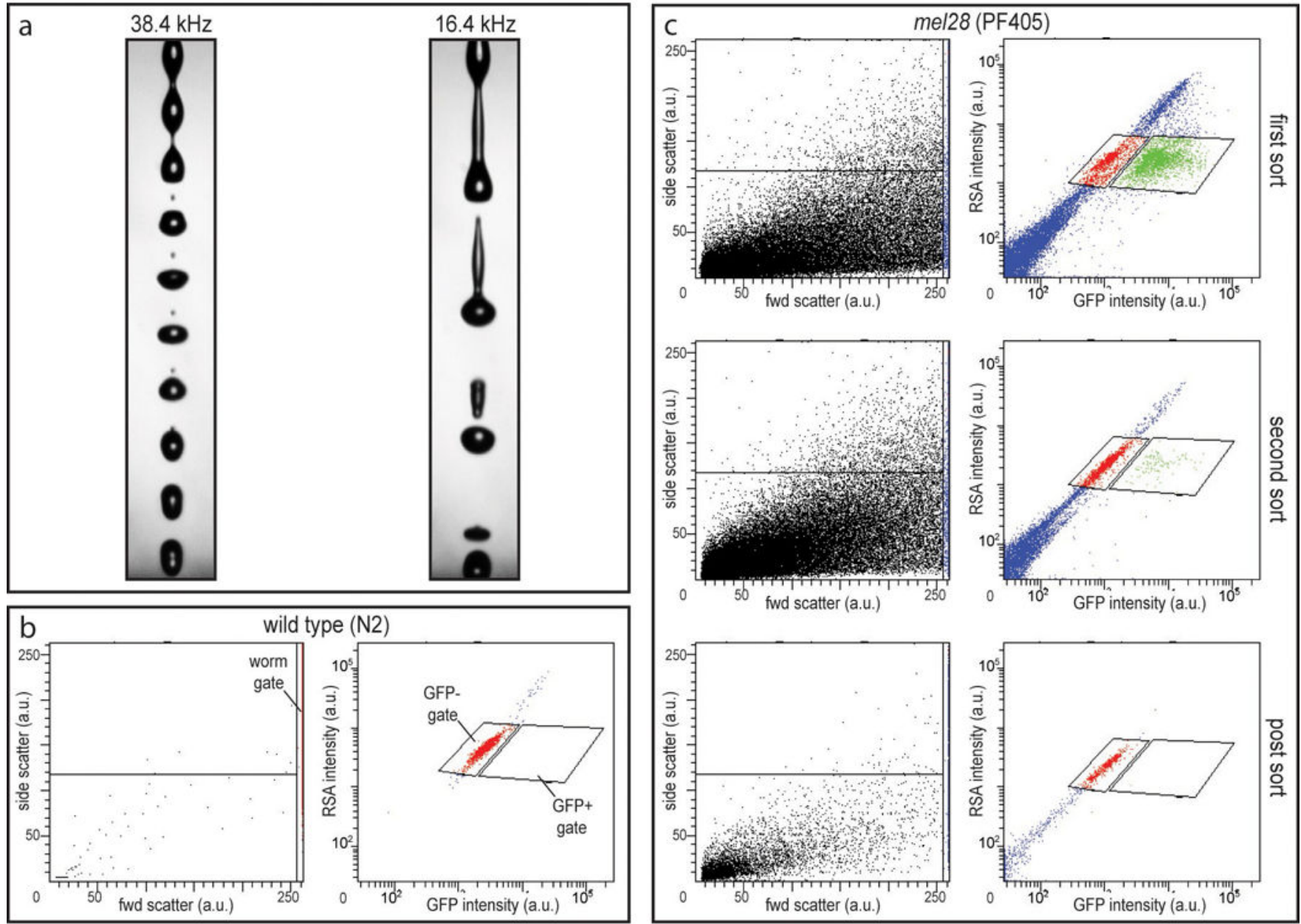

Figure 4. Sorting C. elegans with laFACS

(a) Low-frequency drop-drive stream set up on the FACS facilitates larval survival. (b) Wildtype (N2) L1 larvae were assessed to visualize purely GFP-negative larvae. A gate was set to distinguish larvae from debris in the forward scatter vs. side scatter plot (worm gate; left panel) and two gates were set to identify GFP-negative and GFP-positive events in the GFP intensity (488 nm excitation, 530/30 emission) vs. red spectrum autofluorescence (RSA; 488 $\mathrm{nm}$ excitation, $610 / 20 \mathrm{~nm}$ emission) plot (GFP- and GFP+ gates, respectively; right panel).

(c) Successive sorts showing the purification of GFP-negative larvae from a PF405 L1 population. 


\section{Table 1}

\section{GFP-marked balancers in C. elegans ${ }^{a}$}

\begin{tabular}{|c|c|c|c|c|}
\hline $\begin{array}{l}\text { Name of } \\
\text { Balancer }\end{array}$ & $\begin{array}{l}\text { Homo. } \\
\text { Lethal? }\end{array}$ & Region Balanced & Type of Balancer & GFP Marker \\
\hline$n T 1^{b}[q I s 51]$ & yes & $\begin{array}{l}\text { right end of chromosome IV } \\
\text { through unc-17, left end } \\
\text { chromosome } \mathrm{V} \text { through unc- } 76\end{array}$ & $\begin{array}{l}\text { reciprocal } \\
\text { translocation } c\end{array}$ & $\begin{array}{l}\text { myo2::GFP, } \\
\text { pes-10::GFP, } \\
F 2 B 7.9:: G F P\end{array}$ \\
\hline$h T 2^{d, e}[q I s 48]$ & yes & $\begin{array}{l}\text { chromosome I from left end } \\
\text { through unc-101, chromosome } \\
\text { III from right end through } d p y- \\
17\end{array}$ & $\begin{array}{l}\text { reciprocal } \\
\text { translocation } c\end{array}$ & $\begin{array}{l}\text { myo2::GFP, } \\
\text { pes- } 10:: G F P, \\
\text { ges- } 1:: G F P\end{array}$ \\
\hline$m I n 1^{f}[m I s 14]$ & no & $\begin{array}{l}\text { chromosome II between lin-31 } \\
\text { and rol-1 }\end{array}$ & inversion & $\begin{array}{l}\text { myo2::GFP, } \\
\text { pes-10::GFP, } \\
F 2 B 7.9:: G F P\end{array}$ \\
\hline$q C 1[q I s 26]^{g}$ & yes & $\begin{array}{l}\text { left portion chromosome III } \\
\text { between tra- } 1 \text { and } d p y-1\end{array}$ & unknown & lag-2::GFP \\
\hline eT1[nIs267] & no & $\begin{array}{l}\text { chromosome V left end } \\
\text { through unc- } 23 \text {, chromosome } \\
\text { III right end through unc-36 }\end{array}$ & $\begin{array}{l}\text { reciprocal } \\
\text { translocation } c\end{array}$ & myo-2::GFP \\
\hline$m I s 10^{h}$ & no & $\begin{array}{l}\text { chromosome } \mathrm{V} \text { between unc- } \\
60 \text { and } d p y-11 \text {. }\end{array}$ & insertion & $\begin{array}{l}\text { myo2::GFP, } \\
\text { pes-10::GFP, } \\
F 2 B 7.9:: G F P\end{array}$ \\
\hline$m I s 11$ & no & $\begin{array}{l}\text { chromosome IV, unknown } \\
\text { interval near } d p y-20 \text {. This has } \\
\text { been used successfully to } \\
\text { balance deletions in cyb-1, syn- } \\
4 \text { tag-316, htp-1, mep-1, and } \\
\text { tag-137. }\end{array}$ & insertion & $\begin{array}{l}\text { myo2::GFP, } \\
\text { pes-10::GFP, } \\
F 2 B 7.9:: G F P\end{array}$ \\
\hline mIs12 & no & $\begin{array}{l}\text { chromosome II ,unknown } \\
\text { interval near unc-4. }\end{array}$ & insertion & $\begin{array}{l}\text { myo2::GFP, } \\
\text { pes-10::GFP, } \\
F 2 B 7.9:: G F P\end{array}$ \\
\hline mIs13 & no & $\begin{array}{l}\text { chromosome I, unknown } \\
\text { interval near unc-54. This has } \\
\text { been used successfully to } \\
\text { balance deletions in tag-115, } \\
\text { kin-1, npp-4, ero- } 1 \text { and } b b s-1 \text {. }\end{array}$ & insertion & $\begin{array}{l}\text { myo2::GFP, } \\
\text { pes-10::GFP, } \\
F 2 B 7.9:: G F P\end{array}$ \\
\hline okIs57 & no & $\begin{array}{l}\text { chromosome } \mathrm{X} \text {, unknown } \\
\text { interval near unc-3. }\end{array}$ & insertion & $\begin{array}{l}\text { myo2::GFP, } \\
\text { pes-10::GFP, } \\
F 2 B 7.9:: G F P\end{array}$ \\
\hline okIs59 & no & $\begin{array}{l}\text { chromosome I, unknown } \\
\text { interval between } d p y-5 \text { and } \\
\text { unc-13. This has been used to } \\
\text { balance deletions in air-2, dao- } \\
5, \text { npp-7, chn-1, tag- } 83 \text { and gly- } \\
\text { 2. }\end{array}$ & insertion & $\begin{array}{l}\text { myo2::GFP, } \\
\text { pes-10::GFP, } \\
F 2 B 7.9:: G F P\end{array}$ \\
\hline
\end{tabular}

Information on balancers comes from WS228, 19,20 , and author observations

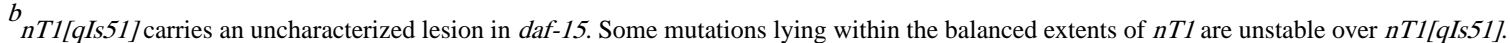

$c_{\text {Reciprocal translocation balancers produce aneuploid inviable progeny }}{ }^{20}$, and this reduces the expected yield of viable homozygotes.

$d_{\text {The }} q I s 48$ element is sometimes lost by apparent rare recombination, leaving behind viable $h T 2$ homozygotes marked with the original bli-4 mutation.

Some mutations lying within the balanced extents of $h T 2$ are unstable over hT2[qIs 48 ].

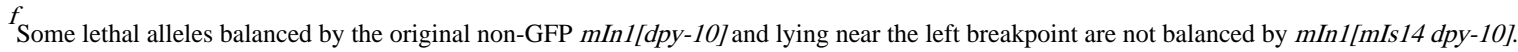

${ }^{g}$ qIs26 also carries dominant rol-6 allele su1006. 


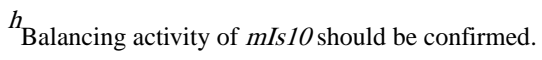

